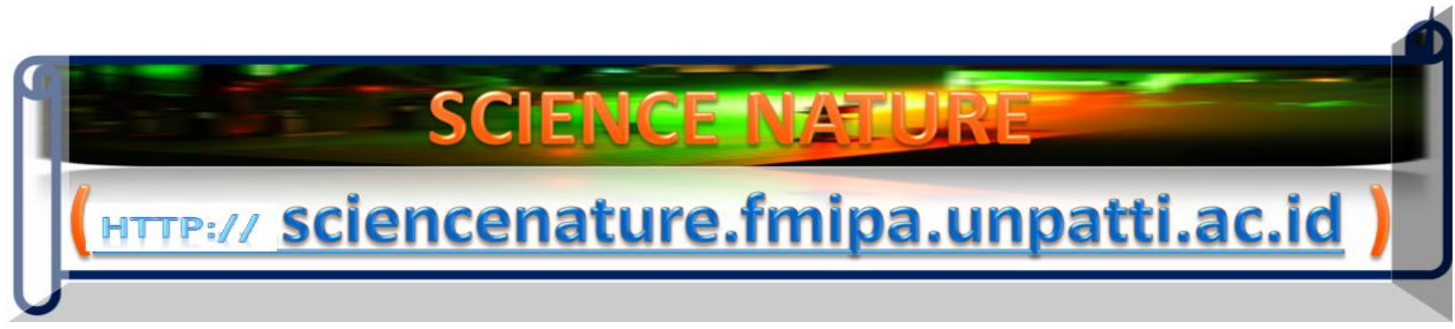

Science Nature 1(1), pp.001-007 (2018)

e-ISSN: 2554-6264

DOI: https://doi.org/10.30598/SNVol1Iss1pp001-007year2018

\title{
Aspect-ratio Dependence of Optical Nonlinearities on Resonance with Longitudinal Surface Plasmon in Au Nanorods: Unique Character versus Common Behavior
}

\author{
Hendry I. Elim ${ }^{\mathbf{1 , 2 3 , 4}} \mathbf{4}^{*}$, Wei $\mathrm{Ji}^{5}$, Mohan Singh Dhoni ${ }^{5}$, N. Venkatram ${ }^{5}$, Jian Yang ${ }^{6}$, \\ and Jim Yang Lee ${ }^{6}$

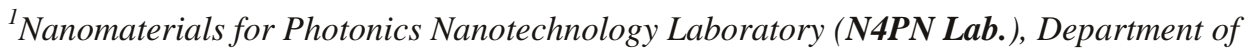 \\ Physics, Faculty of Mathematics and Natural Sciences (FMIPA), Pattimura University (UNPATTI), \\ Jl. Ir. M. Putuhena, Poka, Ambon, Indonesia 97233 \\ ${ }^{2}$ Nanotechnology Research Center and Innovative Creation (PPNRI-LPPM), \\ Research Center of Pattimura University, UNPATTI, \\ Jl. Mr. CHR. Soplanit, Rumah Tiga, Ambon, Indonesia 97234 \\ ${ }^{3}$ Multidisciplinary Research Center of Excellence (MrCE), UNPATTI, Jl. Dr. Leimena, Ambon, Indonesia 97234 \\ ${ }^{4}$ Theoretical Physics Laboratory (TP Lab.), Department of Physics, FMIPA, UNPATTI, \\ Jl. Ir. M. Putuhena, Poka, Ambon, Indonesia 97233 \\ ${ }^{5}$ Department of Physics, 2 Science Drive 3, National University of Singapore, \\ Singapore 117542 \\ ${ }^{6}$ Department of Chemical and Biomolecular Engineering, National University of Singapore, 4 Engineering Drive 3, \\ Singapore 117576
}

Received : July 24, 2018

Revised : July 28, 2018

Published : July 31, 2018

Copyright @ All rights are reserved by Hendry I. Elim

Corresponding author: "Email: hendry.elim@staff.unpatti.ac.id; hendryelim@gmail.com 
Aspect-ratio Dependence of Optical Nonlinearities on Resonance with Longitudinal Surface Plasmon in Au Nanorods: Unique Character versus Common Behavior

\section{Abstract}

Unique character and common behavior are two distinguished things. In frontier nanoscience and nanotechnology, unique characters were normally obtained in some novel exotics materials such as metal-metal core-shell materials, metallic-semiconducting hybrid nanomaterials, and organic-inorganics complex nano-compounds. On the other hand, normal behavior of natural phenomena including in nano-size objects were obviously predicted based on their exact size related to confinement effect, and capability to interact with another physical system in nature. Here, we report an example of unique character due to evolution nonlinear behavior observed in gold nanorod with their aspect-ratio dependence of optical nonlinearities investigated by femtosecond Z-scan measurements closed to resonance longitudinal surface plasmon peak in gold nanorods (Au NRs). Saturable absorption manifests itself at low excitation (laser irradiances $<\sim 7.0 \mathrm{GW} / \mathrm{cm}^{2}$ ), while reverse saturable absorption dominates at higher excitation. Both the nonlinear processes are found to increase with the aspect ratio of $\mathrm{Au}$ NRs. Based on the discrete dipole approximation, qualitative explanations are presented for the observed nonlinear behavior. While common behavior in metallic quantum dots or other shapes of metallic nanomaterials was not the significant in our observation.

Keywords: Unique character, Aspect ratio, Gold nanorod,

Optical nonlinearity.

\section{$\underline{\coprod \text { ETTERS }}$}

Frontier works in nanoscience and nanotechnology currently have paid various multidisciplinary investigations particularly in conjunction with the nonlinear optical (NLO) behaviors in metal nanorods due to their wide impacts in many integrated applications including in advanced photonics devices [1-5]. The normal behaviors of NLO processes in metal nanorods have been well known to be significantly much more improvements in many physical properties then those in the bulk or nanoparticles. Furthermore, recent research

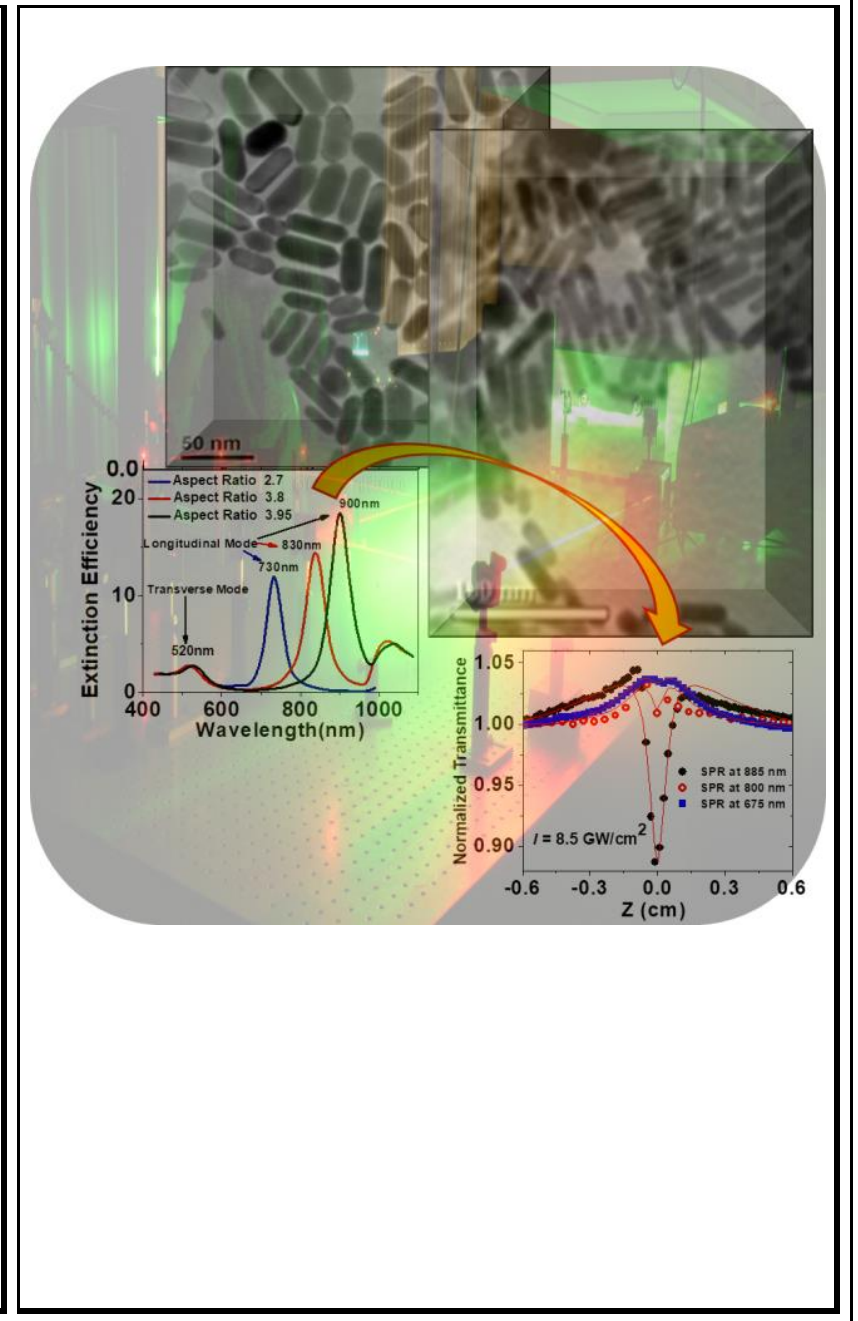

works related to the strong enhancement of emission from gold nanorods (Au NRs) near their surface plasmon resonance (SPR) have made $\mathrm{Au}$ NRs promising for many applications in nanotechnology [6-8]. Surface plasmon consists of electromagnetic waves that oscillate collectively at a metal/dielectric interface. Because of its capability to localize and guide light in sub-wavelength metallic nanostructures, surface-plasmon-based photonics or plasmonics, offers an opportunity to merge photonics and electronics on nanoscale dimensions. Moreover, NLO effects, including saturable absorption and reverse saturable absorption, are also enhanced considerably due to the local-field enhancement occurring at the SPR as a result of large optical 
Aspect-ratio Dependence of Optical Nonlinearities on Resonance with Longitudinal Surface Plasmon in Au Nanorods: Unique Character versus Common Behavior

polarization associated with the collective electron oscillations. As such, there have been many reports on the interesting NLO properties of metal nanoparticles/nanowires that give immense enthusiasm for their applications such as optical limiters, filters, waveguides, lenses, polarizers, sensor protection, biological imaging, medicine, and nanoprobes [9-13].

In this letter, we report a unique character of nonlinear optical phenomena changing due to saturable absorption (SA), and reverse saturable absorption (RSA) which are dependence on both the aspect ratio of $\mathrm{Au}$ NRs and femtosecond laser light intensity at $\sim 700 \mathrm{~nm}$, $800 \mathrm{~nm}$ and $880 \mathrm{~nm}$ wavelengths, respectively. Our investigation into the aspect-ratio-dependent NLO properties of $\mathrm{Au}$ NRs was carried out in such above mentioned wavelengths with the aim of approaching to their longitudinal peak mode of SPR.
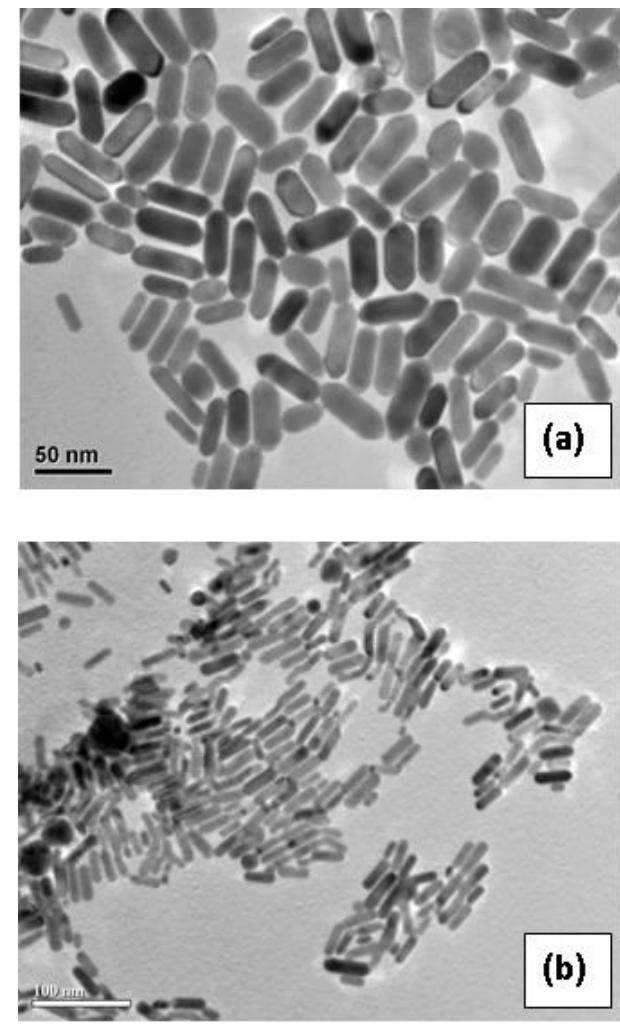

Figure 1. Typical TEM images of Au NRs with aspect ratios of 2.7 (a) and 3.8 (b).
The details of preparing $\mathrm{Au}$ NRs were reported elsewhere [12]. According to the transmission electron microscopic (TEM) images, as displayed in Fig. 1, we obtained the three samples of Au NRs with the aspect ratio as follows: $2.7,3.8$, and 3.95 , respectively. The absorption spectra of the three samples of $\mathrm{Au}$ NRs dispersed in water were measured with an ultraviolet-visible spectrophotometer (UV-1700 Shimadzu). As shown in Fig. 2(a), it is evident that there are two SPR peaks. The first and small SPR peak for all the three samples is located almost at the same wavelength of $520 \mathrm{~nm}$, and it is due to the transverse mode perpendicular to the $\mathrm{Au} \mathrm{NRs}$, (and the average width of all $\mathrm{Au}$ NRs has approximately the same size); and the second and large SPR peak are located at different wavelengths of 675,800 , and $885 \mathrm{~nm}$, and these SPR peaks are due to the longitudinal mode of SPR with the peak position depending on the length of Au NRs.

It is well-known that the longitudinal surface resonance exhibits a larger redshift with a greater aspect ratio (the length divided by the width) of metal nanorods. To gain more insight into the spectra described above, we have carried out the theoretical calculation using the discrete dipole approximation (DDA) method. The DDA method has been regarded as one of the most powerful and flexible electrodynamic methods for computing the extinction coefficient and optical scattering of metallic nanostructures with an arbitrary geometry $[\mathbf{1 4 , 1 5}]$. In our calculation, we have adopted the DDA code developed by Draine and Flatau $[\mathbf{1}, \mathbf{1 6}]$. We first characterize the case with fixed orientation where the propagation direction of the incident light is assumed to be perpendicular to the axis of symmetry (long axis) the nanorod; and then averaged all the directions by considering the random distribution of Au NRs in our experiments. The nanorod is considered to have a geometry being a cylinder capped with two hemispheres. The calculations are carried out for varying aspect ratios of the nanorods. The theoretical results are shown in Fig. 2(b), which is qualitatively 
Aspect-ratio Dependence of Optical Nonlinearities on Resonance with Longitudinal Surface Plasmon in Au Nanorods: Unique Character versus Common Behavior

agreeable to the data in Fig. 2(a). The calculated longitudinal modes have a narrower bandwidth and are red-shifted, compared to the experimental data. The difference may be attributed to the fact there is a finite size dispersion of $\mathrm{Au}$ NRs used in the experiment. It is particularly larger for Au NRs of smaller aspect ratios which have relative larger dispersions in the length.

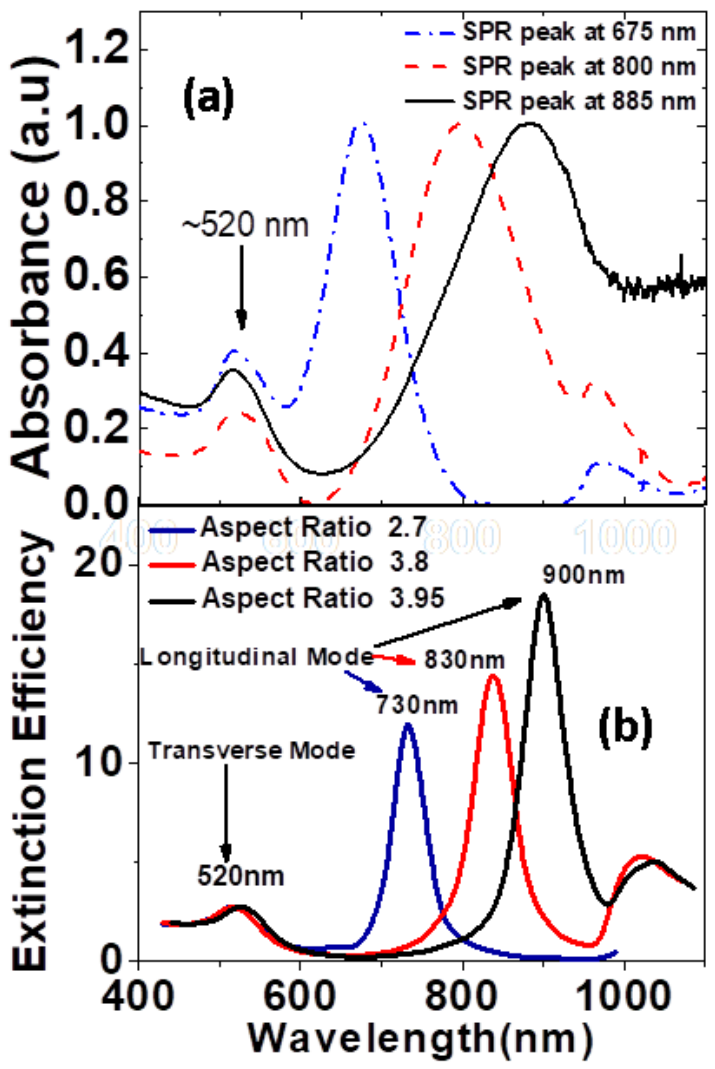

Figure 2. (a) Measured absorbance and (b) theoretical spectra of $\mathrm{Au}$ NRs in water with different aspect ratios. The measured absorbance spectra are normalized to the peak of the longitudinal SPR. The inset in (b) zooms into the weak transverse SPR at $\sim 520 \mathrm{~nm}$.

The longitudinal-SPR NLO properties of Au NRs with the aspect ratio of 3.8 has been studied in detail and reported previously [12]. Here, we focus our attention to the NLO measurements with femtosecond $Z$-scans at laser wavelengths $(\sim 700 \mathrm{~nm}, 800 \mathrm{~nm}$ and $880 \mathrm{~nm})$ near /at the peak positions $(675 \mathrm{~nm}, 800 \mathrm{~nm}$ and $885 \mathrm{~nm})$ of longitudinal SPR for the three samples, respectively. To minimize accumulative thermal effects, we employed 220 -fs laser pulses at $1-\mathrm{kHz}$ repetition rate. The laser pulses were generated by a modelocked Ti: Sapphire laser (Quantronix, IMRA), which seeded a Ti:Sapphire regenerative amplifier (Quantronix, Titan). The wavelengths were tunable as the laser pulses passed through an optical parametric amplifier (Quantronix, TOPAS). The laser pulses were focused onto a 1-mm-thick quartz cuvette which contained the Au NR solution with a minimum beam waist of $10 \mu \mathrm{m}$. By adding water to the $\mathrm{Au} \mathrm{NR}$ solution, the linear transmittance of all the solutions was adjusted to be $\sim 70 \%$ at the laser wavelengths, which kept the same value for the linear absorption coefficient. The incident and transmitted laser powers were monitored as the cuvette was scanned along the $\mathrm{Z}$ direction or the propagation direction of the laser pulses. Figure 3(a) shows typical open-aperture Z-scans for all the Au NR samples performed with the same irradiance of 1.8 $\mathrm{GW} / \mathrm{cm}^{2}$, demonstrating negative signs for the absorptive nonlinearities. We attribute this negativity to saturable absorption (SA), or the bleaching of the ground-state plasmon at the longitudinal mode of SPR due to the excitation of the electrons in Au NRs.

As the excitation irradiance is increased further beyond 7.0 GW/ $\mathrm{cm}^{2}$, as shown in Fig. 3(b), the reverse saturable absorption (RSA) happens due to excited-state absorption (or free-carrier absorption) and nonlinear scattering. To quantify both SA and RSA, we employ the following expression for the entire absorption coefficient of Au NRs in solution:

$\alpha(I)=\frac{\alpha_{0}}{\sqrt{1+I / I_{s}}}+\beta I$,

where $\alpha_{0}$ is the linear absorption coefficient, $I$ is the laser intensity, $I_{s}$ is the saturation intensity, and $\beta$ is the 
Aspect-ratio Dependence of Optical Nonlinearities on Resonance with Longitudinal Surface Plasmon in Au Nanorods: Unique Character versus Common Behavior

nonlinear absorption coefficient which includes excited-state absorption and nonlinear scattering. In the denominator of the second term on the left side of Eqn. (1), the square root is used to reflect the inhomogenous nature of our system since the sizes of Au NRs are not uniform. By applying Eqn. (1) to Z-scan theory, we obtain Z-scan simulations. From the best fittings, we unambiguously extract the two parameters as $I_{s}$ can be determined at lower irradiances and subsequently $\beta$ is obtained at higher irradiance. The extracted values are as follows: $I_{s}=0.08,0.085,0.07 \mathrm{GW} / \mathrm{cm}^{2}$; and $\beta=0.1$, $0.15,0.58 \mathrm{~cm} / \mathrm{GW}$ for the three samples of aspect ratios:

\section{$2.7,3.8$ and 3.95 , respectively.}

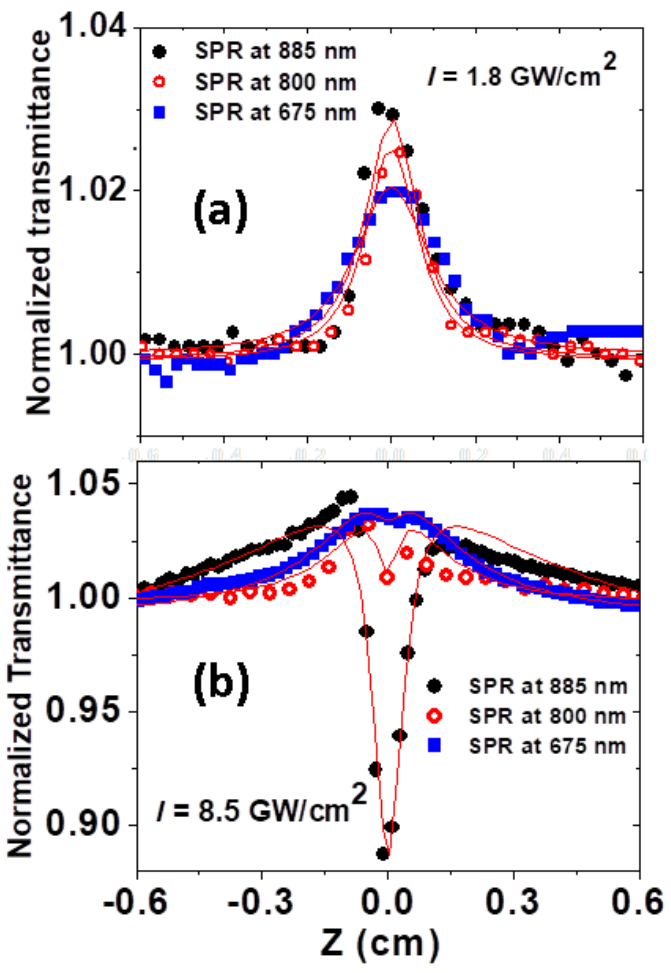

Figure 3. Open-aperture Z-scans (symbols) measured
at (a) $I=1.8 \mathrm{GW} / \mathrm{cm}^{2}$ and (b) $I=8.5$ $\mathrm{GW} / \mathrm{cm}^{2}$ of $\mathrm{Au} \mathrm{NRs}$ with different aspect ratios of $2.7,3.8$, and 3.95 , respectively. The solid curves are the Z-scan simulations by Eqn. (1) in the text.
Within the experimental errors, the values of the measured saturation intensity are independent of the aspect ratio. If one expands the first term on the left side of Eqn. (1), one can approximate the third-order nonlinear absorption coefficient to $\alpha_{2} \approx-\alpha_{0} / 2 I_{\mathrm{s}}$. Since $I_{s}$ is independent of the aspect ratio, then $\alpha_{2}$ is directly determined by $\left|\alpha_{0}\right|$, which is increased with the aspect ratio, as shown in Fig. 2(b) by the qualitative calculation based on the DDA method. As such, one concludes that $\alpha_{2}$ is increased with the aspect ratio. The aspect-ratio dependence of $\beta$ should be anticipated as well since it is induced from light scattering to a large extent. The DDA calculation predicts that the light scattering effect gets stronger as the length of Au NRs increases. As such, the magnitude of nonlinear scattering becomes greater as well.

Finally, based on our scientific measurements and theoretical findings mentioned in the former paragraphs, a remarkable different between normal behaviors and unique character of NLO processes in metal nanorods is not solely on more improvements in physical properties, but also on its evolution nonlinear characters in keep changing as high intensity fs laser changes during light-nm scale matter interactions with them especially on their longitudinal SPR with larger number of surface plasmon electrons than that in their transversal SPR mostly associated with their diameter of rods.

In conclusion, we have observed the evolution nonlinear characters with aspect-ratio dependence of NLO properties in Au NRs with femtosecond Z-scans at/near longitudinal SPR. The nonlinear absorption properties in $\mathrm{Au}$ NRs are found to be dependent on excitation irradiance. Saturable absorption occurs at low laser irradiances $\left(<\sim 7.0 \mathrm{GW} / \mathrm{cm}^{2}\right)$ and its magnitude increases as the aspect ratio increases. Reverse saturable absorption dominates the higher irradiance regime, and it is dependent on the aspect ratio in a similar fashion. The mechanisms responsible for such aspect-ratio dependence are discussed under the discrete dipole 
Aspect-ratio Dependence of Optical Nonlinearities on Resonance with Longitudinal Surface Plasmon in Au Nanorods: Unique Character versus Common Behavior

approximation. While the evolution nonlinear optical characters were observed by changing the fs laser intensity as well as its tunable wavelengths closed to the longitudinal SPR peak.

\section{Acknowledgement}

H.I.E is grateful to thank Prof. Ji Wei and Prof. Lee Jim Yang of National University of Singapore for their guidance and financial supports during this research works.

\section{References}

[1] B.T. Draine and P.J. Flatau, J. Opt. Soc. Am. A 11, 1491 (1994).

[2] H.I. Elim, Wei Ji, Jian Yang,and Jim Yang Lee, Appl. Phys. Lett. 92, 251106 (2008).

[3] Jian Yang, H.I. Elim, QingBo Zhang, Jim Yang Lee, and Wei Ji, J. AM. CHEM. SOC. 128, 11921-11926 (2006).

[4] H.I. Elim, Y.W. Zhu, and C.H. Sow, J. Phys. Chem. C 120(31), 17733-17738 (2016).

[5] H. I. Elim, Bin Cai, Okihiro Sugihara, Toshikuni Kaino, and T. Adschiri, Phys. Chem. Chem. Phys 13 (10), 4470 - 4475 (2011).
[6] Y.Y. Yu, S.S. Chang, C.L. Lee and C.R.C. Wang, J. Phys. Chem. B 101, 6661 (1997).

[7] Y. Li and M.A. El-Sayed, J. Phys. Chem. B 105, 8938 (2001).

[8] M.B. Mohamed, V. Volkov, S. Link and M.A. El-Sayed, Chem. Phys. Lett. 317, 517 (2000).

[9] R. West, Y. Wang and T. Goodson III, J. Phys. Chem. B 107, 3419 (2003).

[10] B.K. Pong, H.I. Elim, J.X. Chong, W. Ji, B.L. Trout and J.Y. Lee, J. Phys. Chem. C 111, 6281 (2007).

[11] A.N. Shipway, E. Katz and I. Willner, Chem. Phys. Chem. 1, 18 (2000).

[12] H.I. Elim, J. Yang, J.Y. Lee, J. Mi and W. Ji, Appl. Phys. Lett. 88, 083107 (2006).

[13] H.F. Wang, T.B. Huff, D.A. Zweifel, W. He, P.S. Low, A. Wei and J.X. Cheng, Proc. Natl. Acad. Sc. USA 102, 15752 (2005).

[14] L. Francois, M. Mostafavi, J. Belloni and J.A. Delaire, Phys. Chem. Chem. Phys. 3, 4965 (2001).

[15] T. Jensen; L. Kelly, A. Lazarides, and G. C. Schatz, J. Cluster Sci. 10, 295 (1999).

[16] Draine, B.T., and Flatau, P.J. 2008, User Guide for the Discrete Dipole, Approximation Code DDSCAT 7.0, http://arxiv.org/abs/0809.0337v4.

\section{${ }^{\star}$ Corresponding Author Brief CV

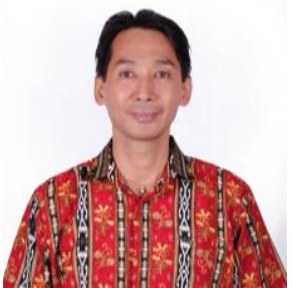

HENDRY IZAAC ELIM, PH.D: Young Scientist 2017 of Pattimura University based on Rector letter dated on 13rd November 2017, No.1157/UN13/SK/2017 (Ph.D.-Physics; personal website: http://fisika.fmipa.unpatti.ac.id/hendry-izaac-elim ), now is an experience Indonesia scientist of nanoscience and nanotechnology (rank 23 $3^{\text {rd }}$ in 2017 based on Webomatrics (http://www.webometrics.info/), and the 45 best Indonesia scientist according to Science and Technology Index (SINTA-RistekDikti in May 2018), head of the Nanotechnology Research Center and Innovative Creation (PPNRI-LPPM, website: http://ppm.unpatti.ac.id/pusat-pnri ) of the Pattimura University (UNPATTI), Chairman for Nanomaterials for 
Photonics Nanotechnology Laboratory (N4PN Lab), Physics Department, Faculty of Mathematics and Natural Sciences (FMIPA-UNPATTI), Ambon, Indonesia, and regular Member of the Indonesia Theoretical Physicist. He got his B.Sc (S.Si). in Theoretical Physics in 1995 at Gadjah Mada university (UGM), the oldest university in Indonesia, M.Si (M.Sc). in Theoretical Physics of Institut Teknologi Bandung (ITB) in 1999. Specialist in nanoscience and nanotechnology, Physics Doctor's degree (Ph.D.) at National University of Singapore (NUS), Singapore on $13^{\text {th }}$ December 2005, Docent at FMIPA-UNPATTI since 2000 up to present. After his PhD at NUS, Dr. Elim worked as a postdoctoral fellow in physics department of NUS, and about 2 years later He moved to Tohoku university, Sendai, Japan working on superhybrid materials project at Institute of Multidisciplinary Research for Advanced Materials (IMRAM) from 2007 to 2012. In 2013, Dr. Elim worked as a scientist at Surya university, Indonesia for 3 months and then moved to STKIP Surya, Gading Serpong, Tangerang, Indonesia working as a physics lecturer for 1 year. Later in September 2014, Dr. Elim returned to FMIPA-UNPATTI and started building N4PN Lab as well as PPNRI-LPPM until present time. The advancement of Science and technology development of Dr. Elim group started by educating the first 6 research B.Sc students and since that He already educated more than 50 graduated B.Sc in physics from all simple advanced research on novel superfibers fabricated from all types of garbage materials, new liquid energy fuel cells system, and nanomedicine of herbal drugs, respectively. The studies involved their mechanical, optical, and chemical properties as well as molecular electronic system. Ricently. Dr. Elim is leading research on water contaminated by $\mathrm{CaCO}_{3}$, the aggregation of salt behaviors in ocean water and energy research development and their relationships with herbal medicine extracted from fruits and leaves. Furthermore, Dr. Elim educated few research students to work on bio-membrane films and fibers fabricated using rubbish natural things. These studies involved their mechanical and optical behaviors. In addition, Dr. Elim had been recently invited to give international scientific talks as well as many quest lecture talks in many national universities such as UNPATTI, UNIMA, UNSRAT, STIKES Halmahera, AKBID MAKARIWO, and UI of Indonesia closely related to the multitasking applications of nanoscience and nanotechnology in small islands and human characters. In addition, Dr. Elim was invited to give a talk in a breakthrough international conference of pharmaceutical nanomedicine and nanotechnology in Las Vegas, USA in April 2018. Based on the international community data recorded in Web of Science, Dr. Elim have published over 50 papers with h-index of 23, and citation more than 2000. In addition, Dr. Elim is a member of top scientific society such as AAAS (ID No. 41737449) since 2017, and ACS (ID No. 31447106) in 2018. Dr. Elim was also awarded Saryalancana Karya Satya XX from President of Indonesia on $2^{\text {nd }}$ May 2017 at Pattimura university.

ORCID ID: 0000-0002-4272-7115

URL: http://fisika.fmipa.unpatti.ac.id/hielim

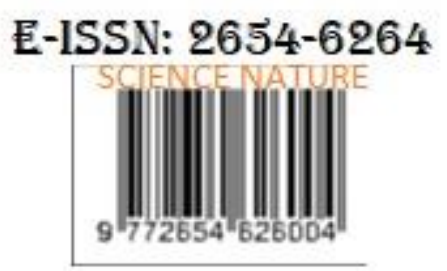

